\title{
Nonanimal Approaches to Assessing the Toxicity of Inhaled Substances: Current Progress and Future Promise
}

\author{
Moderator: Amy J. Clippinger ${ }^{1}$ \\ Participants: David Allen, Holger Behrsing, Paul M. Hinderliter, \\ Robert Landsiedel, Emily N. Reinke, and Vicki Stone ${ }^{7}$
}

\section{Introduction}

A N EXPERT ROUNDTABLE discussion was convened on the development and implementation of 21 st century nonanimal approaches to assess the toxicity of inhaled substances. The following discussion includes perspectives from government, industry, nonprofit, and academic sectors on the advantages and challenges of using these approaches, ongoing efforts, and steps needed to gain widespread use and acceptance of these approaches.

Dr. Amy Clippinger: Thank you all for joining this panel discussion. To begin: What are the most promising in vitro and in silico methods to assess the toxicity of inhaled materials?

Dr. David Allen: As far as the most exciting methods under development to assess the toxicity of inhaled materials, certainly many of the microphysiological systems under developmentwhich, in many respects, mimic the biology and the physiology of the respiratory tract-are being used to generate some very impressive data. I think the jury is still out on the practicality of those approaches in a routine toxicity testing paradigm, but it is very exciting to see the developments underway.

For immediate-term use, commercially available threedimensional airway epithelial models include, depending on the model, different respiratory cell types and are very useful for assessing portal-of-entry effects in the respiratory tract. There are also precision-cut human lung slices, which include multiple cell types retained in an in vitro setting to closely mimic the organ in situ.

From an in silico aspect, there is a lot of effort going into building quantitative structure-activity relationship approaches for other routes of entry. I think the inhalation route needs ad- ditional attention, and certainly there are many opportunities for further development. Computer-based approaches such as three-dimensional computational fluid dynamic (CFD) models are being used now to obtain information one might be concerned with regard to exposure, such as where a substance might deposit in the respiratory tract.

Dr. Holger Behrsing: When it comes to the different nonanimal in vitro methods, we break it down into two buckets. One is more for screening efforts where you are trying to weed out the poor players - those that may have adverse effects at concentrations or exposures wherein you really do not want to have that. For these screening efforts that mainly compare the toxicities of the different materials, there are a number of two-dimensional systems that may work quite well and are economically feasible.

For high-content testing of a smaller number of materials, you want to use most physiologically relevant models out there because we want to try to recapitulate, as much as possible, the human respiratory tract. These models include the three-dimensional reconstructed human tissue models and lung slices. The various models will have a different complement of cell types, including immune-competent cell types, which allow us to paint a much clearer picture of what may happen in vivo.

Dr. Robert Landsiedel: I would add macrophage assays to what is immediately usable when it comes to testing particles.

In addition to in vitro and in silico methods, we should add physicochemical methods, because some, if not many, of the effects in the respiratory tract are simple physical interactions. For example, you do not need living cells to study clumping of materials or disturbance of surfactant filmsyou can simply look at physicochemical interactions.

\footnotetext{
${ }^{1}$ Director, PETA International Science Consortium Ltd., London, United Kingdom.

${ }^{2}$ President-Science and Strategy, Integrated Laboratory Systems, Morrisville, North Carolina.

${ }^{3}$ Principal Scientist, Institute for In Vitro Sciences, Inc., Gaithersburg, Maryland.

${ }^{4}$ Senior Scientist, Syngenta Crop Protection, Inc., Greensboro, North Carolina.

${ }^{5}$ Vice President, Experimental Toxicology and Ecology, BASF SE, Ludwigshafen am Rhein, Germany.

${ }^{6}$ Biologist-PI, U.S. Army Public Health Center, Aberdeen Proving Ground, Aberdeen, Maryland.

${ }^{7}$ Director of the Institute of Biological Chemistry, Biophysics and Bioengineering, Heriot-Watt University, Edinburgh, United Kingdom.
}

(C) Amy J. Clippinger et al., 2018; Published by Mary Ann Liebert, Inc. This Open Access article is distributed under the terms of the Creative Commons License (http://creativecommons.org/licenses/by/4.0), which permits unrestricted use, distribution, and reproduction in any medium, provided the original work is properly cited. 
And I would reiterate Dave's point that in silico models are useful to find out where particles deposit, but we need to add information on how long they stay there.

Dr. Vicki Stone: Something that I am quite interested in—due to a European project called PATROLS (Physiologically Anchored Tools for Realistic nanOmateriaL hazard aSsessment) - is the ability to extend the duration of in vitro experiments beyond a couple of days. The PATROLS project includes a threedimensional lung coculture system prolonged, at the moment, for up to 14 days, but aiming for 21 days. That may be with or without some fluidics in the basolateral compartment, and perhaps with or without some flexing of the membranes. Similar work has been done on a chip, but at a fairly small scale in terms of size, which makes the endpoint analysis difficult because there is a lack of sensitivity of the endpoint analysis that is available currently. The PATROLS project will do it at a slightly larger milliliter scale rather than the laboratory-on-a-chip scale, and really extend the duration of the experiment.

Dr. Paul Hinderliter: One thing that we have been excited about at Syngenta for in vitro and in silico methods is the convergence of these methods that allows us to look at risk identification instead of only hazard identification. The in vitro technologies give us a chance to identify hazards and potential effects, which answers part of our question. This, when combined with in silico techniques, allows us to define what the risk is.

And, this convergence allows us to address our goal to reduce animal usage because much animal testing occurs only for identifying hazard. But how do we manage risk? How do we include exposure information? How do we do a full source to outcome assessment of what the risk actually is from inhaled materials?

Dr. Clippinger: Building more specifically on some of the methods that were just mentioned, does anyone have examples of how your organization is applying these methods today or will be in the near future?

Dr. Reinke: What we are primarily doing in our laboratory now is looking to identify and eliminate toxic chemicals during the research and development phase before they make it to production. In the early stages of development, with consistent communication with the developers, is where the in silico and early high-throughput in vitro approaches really can do a good job of making sure that substances we are developing are the least harmful to the soldiers, civilians, and the environment.

Dr. Stone: I would like to pick up on the macrophage model that Robert mentioned. We are in the process of starting to put together some intelligent testing strategies, or integrated approaches to testing and assessment (IATAs), and the macrophage assay will play a key early assessment role in tiers of these strategies because they have a well-established role in the pathogenicity of fibers in the body.

Dr. Behrsing: There are many different ways to recreate inhalation-like exposure in vitro. Now, we have these fantastic three-dimensional human tissue models, and we have equipment that will allow us to not only expose them in an inhalation-like manner, but also make pretty decent assessments of what those tissues are exposed to-dealing with the mass of material that is deposited, the particle size, and even the chemistries that get to that site of exposure. I think all of these new technologies are going to work together, just like in silico and in vitro.

Dr. Landsiedel: We are testing very different substances, ranging from fibers to solid and liquid particles to vapors. It is very difficult to find one method or a general testing scheme to cover it all by the same methodology.

We can be successful finding similarities or differences between materials if we have groups of substances of which we know something about their inhalation toxicity, the target structures, and the effects and the modes of action. ${ }^{1,2}$ If we have a substance group, then we are able to pick an in vitro method that is good in testing this group of materials and even rank them by potency. We do that for polymers, particles, and for specific vapors. At the moment, the testing scheme is tailor-made for a specific problem and then applied to a group of substances.

I would add again the physicochemical methods, which have helped us a lot, for specific methods like surfactant disturbance. For particles, we often use the in vitro macrophage assay, and for vapors and also polymers, we often use precision-cut lung slices.

Dr. Clippinger: When you talk about this macrophage assay, are you in particular talking about the rat cell line assay that you published on? ${ }^{3}$ Or is there other ongoing work to develop a human cell line-based macrophage assay?

Dr. Stone: It is really quite simple to use peripheral blood mononuclear cells from humans. But, the problem with them is you get a lot of variability from person to person, and probably even within a person. So, we tend to find that there are a couple of good macrophage cell lines. A mouse macrophage cell line called J774 is very good. We find it to be quite predictive of the peripheral blood mononuclear cells. Rat NR8383 alveolar macrophages also work well for quite a wide variety of materials; they are a bit more robust and easier to reproduce than the primary cells.

There is a human cell line called the THP-1, but it is actually extremely variable — even within one laboratory, not just across the laboratories-because it is quite an immature cell, and you have to go through many steps to differentiate it. That differentiation process tends to induce quite a lot of variability in that cell line.

Dr. Landsiedel: I agree with Vicki on the increased variation from cells that need to differentiate. Blood mononuclear cells cause quite a lot of variability, and that is one reason why we use the rat or other stable cell lines. I think for some mechanisms at which we are looking now, the difference between species is probably not that relevant.

Dr. Hinderliter: One thing to add, in the agrochemical area, we have been a little bit late at including the impact of physical state on the inhalation dosimetry question. When designing formulations and products, physical state and formulation ingredients have always been part of the design process to give 
us the properties that we want for our products; however, the implications of these for toxicity have been recently examined. What some of these in vitro and in silico models allow us to do is screen ingredients so that we are not adding components to our products that increase toxicity after inhalation exposure, and to understand our products in inhalation exposure pathways-where they would go and what they would do.

\section{Dr. Clippinger: Paul, are you able to share any informa- tion about the approach that you presented at the Society of Toxicology conference last year?}

Dr. Hinderliter: Sure. We have an ongoing project that we have been sharing with the U.S. EPA Office of Pesticide Programs for a pesticide registration where we have been advocating for a source-to-outcome approach. In this approach, we combine all of the different data streams available to us, starting with exposure information. We have been moving from passive exposure information, which is concentration, but not physical state, to including aerosols or particle sizes. Combining exposure with in vitro measures of toxicity and an in silico dosimetry model, either the multiple-path particle dosimetry model or an advanced CFD model from Rick Corley's group at Pacific Northwest National Laboratory (PNNL) gives us a way to put all of our inhalation data together and provide a full risk assessment, as opposed to only a hazard identification. In conjunction, we are using the Epithelix MucilAir ${ }^{\mathrm{TM}}$ human tissue model, which gives us a human-based in vitro endpoint that is as good as or better than an endpoint from an in vivo rodent test to give us a full picture of the inhalation risk that our products pose. We have been discussing this approach with the U.S. EPA for a few years now and, in particular, the risk assessment for a couple of our compounds. A Science Advisory Panel meeting on this topic is likely in October 2018.

Dr. Stone: Is there any data to show that the Epithelix MucilAir model is better than the rodent model? How do you define that?

Dr. Hinderliter: It comes back to what we are actually looking to measure. For the chemicals that we have been doing an assessment on, we have been looking at irritation endpoints rather than more specific types of mechanisms. The threedimensional reconstructed human tissue model, MucilAir, has been shown to be very effective for this type of endpoint. The model measures membrane integrity, cellular function, metabolic function, and cell viability, so we get a good cross section of endpoints that would give us indications of whether or not this type of toxicity is occurring. Because it comprises human cells, it gives us a better dose-response continuum than we were able to get out of inhalation tests in animals. It gives us a more relevant system, and it gives us a better dose-response as we can test more exposure levels.

We have been working to develop an adverse outcome pathway (AOP) framework, and then looking for what steps actually occur leading toward the respiratory tract toxicity that we have observed. For the MucilAir system, the cells are viable for a sufficient length of time that we can see whether we are getting the first events in the irritation pathway. So although we do not track it as long as a subchronic study, we are confident that we have a long enough exposure that we would be able to see the first events in this pathway.
Dr. Landsiedel: You mentioned a better risk estimate than from animal data, which would imply that you have human data to compare with, which usually for pesticides you do not. By "better," do you mean more data?

Dr. Hinderliter: You are correct that we do not have human data available. For ethical and legal considerations, we cannot do human exposures. But, I do find it to be a tougher argument that the findings from a rat test would be more relevant than those from a human-based system. For an inhalation exposure scenario, we have a much more difficult time controlling the atmospheres for the animal exposure and for our in vitro dose-response, we had a dozen dose levels, whereas for the intact animal test, we only had three. So we have a better characterization of what our exposures were in vitro.

Dr. Clippinger: Next, let us look at the advantages and limitations that these nonanimal approaches might be able to offer. Are there advantages that the nonanimal methods can offer that traditional animal inhalation tests do not?

Dr. Behrsing: Certainly, I think that everyone agrees that just having a human-relevant tissue type available is huge because you do not need to try to translate across species. Another benefit that is unique to in vitro systems is that you can control the site of exposure, and you can have different exposure concentrations at that site. Being able to control the environment where you want to evaluate exposure is very helpful, and especially with the lung, where you have this airway continuum where the cells are changing all the way down to the deep lung, and also the deposition is changing as you move deeper in the lung. It is really complex.

Also, some of the models touched on earlier, for example, the CFD model from Rick Corley's group, are pivotal, and can be used to inform what cell types you should be testing using your in vitro system. For example, in addition to MucilAir, Epithelix also makes the SmallAir ${ }^{\mathrm{TM}}$ model, which represents the respiratory bronchioles much further down the respiratory tract. Being able to computationally model what substances should be able to get there after inhalation exposure, and also having an in vitro human cell model that can represent that area of the lung, is really important.

Dr. Reinke: To follow on what Paul was saying, the nonanimal approaches give you the flexibility to test exactly what you need to do. They also have the advantage of repeatability: the $\mathrm{LC}_{50}$ values from animal tests can widely vary each time you conduct the test based on who is doing it, the strain of animal used, and other factors. The nonanimal approaches allow you to repeat an experiment multiple times and show exactly how the chemical reacts with biological endpoints, and somebody on the other side of the world can do it, and you can have confidence that they are most likely going to get the same answer. That is important, because it provides more confidence in the models that you are using to show, yes, we really are getting the right answer.

Dr. Stone: There are several reasons the in vitro models are useful, some of which we have already mentioned. But I also like that you can use these in vitro models for quite an in- 
depth mechanistic understanding sometimes of cellular responses to treatments. That is much more difficult to do in an animal test.

Dr. Landsiedel: I would stress the advantage of being able to repeat experiments, as mentioned before. You can do many more measurements, which is not something you can do in animals.

When testing particles, we have to consider the particle size. If humans are exposed to aerosols with a wide particle size distribution and particles that agglomerate, only the smaller particles or agglomerates reach the bronchi or alveoli. In vitro, we can test the same particle size that humans would be exposed to, but particles should be separated by size to make sure only those that would reach the alveolar region are tested in in vitro models of the lower respiratory tract. In contrast, when testing in vivo, you may have to scale down the size of the particles to account for the smaller size of airways compared with those in humans. This is because particles that can reach the deep lungs of humans may be too large to reach the deep lungs of mice or rats. It can be a technical challenge to generate aerosols in the right particle size range.

Dr. Hinderliter: In addition to increased reproducibility and avoiding interspecies differences, another area that we appreciate the in vitro and in silico tools is that they are very helpful for testing things that we cannot otherwise test. These methods allow us to test different exposure scenarios, different breathing rates, different particle sizes-all of the variables that can go into a system-and even interindividual variability. Those things can be tested with a combined in vitro/in silico technique, and would not be possible to do from just a standard in vivo test.

Dr. Allen: The nonanimal approaches offer increased efficiency, are potentially faster and more cost-effective, and allow the opportunity for higher throughput or higher content formats than is provided by the in vivo test.

\section{Dr. Clippinger: The flip side of that is the next question: What are the limitations that need to be overcome to gain confidence in the use of these nonanimal approaches?}

Dr. Reinke: I think the first thing to overcome is an inertia, both individually and intuitionally, to accepting new approaches. It is the "This is how we have always done it" mindset. That inertia to not want to change approaches or techniques is one of the biggest barriers we are going to have to overcome, but we need to ask whether an approach works well enough, or whether there is a better approach? We know that animal tests are not $100 \%$ reliable either, so let us continue to refine the new upcoming approaches for the best outcomes.

Dr. Behrsing: One thing that I have tried to focus on in working with different in vitro systems is collaborating closely with a pathologist; for example, on the precision-cut lung slice work, a pathologist was pivotal in helping guide the endpoints that we look at. Once you can recreate, using an in vitro system, some of the endpoints that you see in vivo, that is when those naysayers are going to connect the dots and say, "Wait a minute, there might be something here."
One in vitro system cannot reproduce everything that happens in vivo, but AOPs can be used to design an in vitro testing strategy and to determine which in vitro assays can query specific key events in the AOP. There has to be some level of signaling mediation that you can achieve using in vitro, which is in parallel with what you would see in vivo. When you can draw correlations-for example, to a threedimensional system wherein you look at some effects that are further downstream after weeks in culture-then, you start to gain acceptance.

Dr. Stone: Many people conduct testing to comply with regulations, and many of the regulations refer to animal testing. Although there was a movement toward accepting all different types of data available, toxicology is a relatively conservative discipline, because the risks are potentially high. So it is about having the evidence base on which to make a decision, and that evidence base includes the validity of the model that you are using.

Dr. Landsiedel: I would add two technical limitations to acceptance. Usually in vitro models do not have a complete airflow, like we have in the lungs, so often we do not know how much is getting where in the lung. In silico is a promising start to overcome this, especially particle deposition models.

And the other is what Holger mentioned. We have a continuum of different cells from the nose to the alveoli, and with that we have different modes of actions or effects. If you look at respiratory sensitization or obstructive airway diseases or nervous-stimulated effects, it is a wide spectrum of different effects. Naturally, one in vitro model can only address a very limited number of effects, if not only one. Even if you have a good model or combine several good models, you may still miss potential structures and effects that are just not covered by your models. For regulatory acceptance, the question is a framework question: What do you really demand from in vitro models? Do you demand everything an in vivo model is able to provide? Complete reproduction of the in vivo model by in vitro models will be almost impossible. Or would we rather adapt the regulatory needs to in vitro models and define what has to be tested and what not? Then we can go ahead.

Dr. Stone: Even if complete reproduction was possible, it would be quite time consuming and very expensive. People talk about the expense of animal studies. But if you wanted a whole battery of in vitro tests that were going to cover all of the different endpoints you can get from an animal study, that probably eventually would be quite expensive, too.

Dr. Reinke: Another thing to consider is the applicability of the models to a unique set of chemicals. For example, in the Department of Defense, we currently are looking at newly developed chemicals that are used in explosive, smoke, pyrotechnic, and propellant formulations. Even a traditional in vivo exposure scenario can provide unique challenges, such as exposing animals to smoke that is being produced from a live smoke grenade.

When you are trying to use an in silico model, those unique types of chemicals are not necessarily being used to develop the models, so you have a very low confidence in whether those models are going to be applicable to your 
testing space. Something that we are going to have to look at going forward is making sure that the toxicity data from chemicals that are unique are included in those models.

Dr. Stone: If you look at the pattern of what we are discussing, what we have all clearly agreed on is that the in vitro tests have great potential to be used now for screening. In terms of the more complex risk assessment decision-making, that is where the limitations arise, because it is difficult to replicate the complexity of the full physiological system.

Dr. Clippinger: And combining in silico and in vitro methods, as we mentioned, is potentially a way to get around that limitation, and including some of the stillemerging technologies like lung chips. Also, there should be a continued discussion with regulators about what the data needs really are because, as Robert mentioned, whether we are trying to exactly reproduce what is seen in vivo or looking for an alternative testing paradigm that provides sufficient data to protect human health is a critical point. It is likely that we do not need to exactly model what happens in animals, and that we can actually produce more human-relevant results by using different approaches going forward.

Dr. Stone: Something that we are doing at the moment for nanomaterials, within the European project called GRACIOUS (Grouping, Read-Across, CharacterIsation and classificatiOn framework for regUlatory risk assessment of manufactured nanomaterials and Safer design of nanoenabled products), is developing intelligent testing strategies or IATAs so that you can do a risk assessment based on grouping and read-across. And the project does exactly what you have just described: it starts off with physicochemical characteristics, simple in vitro models, and then more complex in vitro and in silico models, and then if required, you go to an animal. It is a tiered testing strategy that brings all existing methods and information together.

Dr. Reinke: I think clarifying the purpose of the study or the regulatory need and then defining those tiers are what really needs to be done now. The testing approach used will not be the same across the board, it will vary depending on the substance you are interested in.

Dr. Stone: Yes, framing is an important component at the beginning of deciding your intelligent testing strategy. What are the most appropriate regulations? What is the most appropriate form of exposure? What is the most appropriate route of exposure? At the beginning, you frame the question and that helps to identify what the testing strategy should be.

Dr. Hinderliter: One thing I would add is that a common problem in toxicology that we run into is putting things in context-for example, when we have done a high-dose animal study and are trying to determine what the relevance of that is to a human exposed under different conditions. We generally take the approach of trying to adapt the problem to the question being asked rather than trying to make the data fit backwards to it. We are not trying to reproduce everything that a rodent might tell you, because that might not be the relevant question.
Dr. Clippinger: Moving on to the next question. What are some of the ongoing efforts to develop, validate, and implement nonanimal approaches for inhalation toxicity? I know you are all involved in major efforts. Vicki, you mentioned the PATROLS and GRACIOUS projects. Robert, I believe you participate in the AeroSafe project. Dave, you have been involved in the acute inhalation toxicity working groups, and Holger, next-generation nicotine product work. Let us start there.

Dr. Allen: Yes, the Interagency Coordinating Committee on the Validation of Alternative Toxicological Methods (ICCVAM) has developed an implementation plan to prioritize the execution of activities to support the development, evaluation, and regulatory use of alternative approaches for acute systemic toxicity resulting from oral, dermal, or inhalation exposure. ${ }^{4}$

This plan provides a strategy for the reduction and replacement of animal use for toxicity testing in several key endeavors: (1) coordinating activities through the ICCVAM Acute Toxicity Workgroup, which comprises experts from the ICCVAM agencies that require or use acute toxicity information; (2) identifying U.S. agency requirements and needs for acute systemic toxicity data; (3) coordinating efforts with stakeholders; (4) identifying, acquiring, and curating high-quality data from reference test methods as well as potential nonanimal alternatives (see https://ice.ntp.niehs.nih.gov/); (5) identifying and evaluating alternative approaches to acute systemic toxicity testing in animals; and (6) gaining regulatory acceptance and use of nonanimal approaches. Successful implementation will necessitate close communication and cooperation with relevant stakeholders through public-private partnerships that include both regulators and the regulated community.

An excellent example of such a collaborative effort was a 2016 meeting, organized by the NTP Interagency Center for the Evaluation of Alternative Toxicology Methods and the PETA International Science Consortium Ltd., which was summarized in the publication "Alternative approaches for acute inhalation toxicity testing to address global regulatory and non-regulatory data requirements: An international workshop report." 5 The workshop was organized in response to interest in the development, validation, and implementation of effective and efficient approaches that replace animal use for acute inhalation toxicity testing, and was attended by about 50 scientists from regulatory agencies, academia, nongovernmental organizations (NGOs), and industry. The product of this workshop was a strategy to optimize these approaches, with four primary recommendations on how to execute this strategy. After the workshop, groups were established to address each of these four recommendations.

Discussions among these working groups are ongoing and anyone interested in joining can contact me or Amy. As a result of these discussions, two additional articles have been drafted: "Status of acute systemic toxicity testing requirements and data uses by U.S. regulatory agencies" 6 and "Pathway-based predictive approaches for non-animal assessment of acute inhalation hazard determination," (Clippinger et al., unpublished data).

Dr. Behrsing: In 2014, the Institute for In Vitro Sciences (IIVS) held an informational workshop, "Assessment of In Vitro COPD Models for Tobacco Regulatory Science", to review nonanimal respiratory toxicology assays, and 
breakout group discussions identified three endpoints—goblet cell hyperplasia, mucus production, and ciliary beat frequency—as having merit for further review. ${ }^{7}$

To facilitate the review of these assays, IIVS has been managing a technical workshop where multiple laboratories are using common protocols to assess the consistency and accuracy of the assays used to measure these endpoints. This ongoing effort allows the refinement of protocols with the ultimate goal of establishing successful methods that allow reproducible results to be generated across different laboratories. This international exercise is currently poised to begin Phase III where inhalation-like exposures will be applied to reconstructed airway tissues grown at the air-liquid interface. Successful implementation and demonstration of reproducible results will position one or more assays for consideration in prevalidation exercises.

Dr. Stone: The PATROLS project kicked off in January 2018 (https://www.patrols-h2020.eu). PATROLS is a Horizon 2020 project combining a team of academics, industrial and NGO scientists, government officials, and risk assessors to deliver advanced and realistic tools and methods for nanomaterial safety assessment. One of the aims of this project is to develop more predictive in vitro three-dimensional respiratory models. Also kicked off in January 2018 is the Horizon 2020 GRACIOUS project. This project brings together 23 partners spanning Europe and the United States and aims to develop an innovative science-based framework that supports the assessment of risk posed by the ever increasing array of nanomaterials on the market (https://www.cordis.europa.eu/project/rcn/ 212339_en.html). The framework will streamline the process for assessing risk by logically grouping nanomaterials, allowing extrapolation (read-across) between nanomaterials, and reducing the need to assess exposure and toxicity on a case-by-case basis.

Dr. Landsiedel: We are participating in the European Union projects PATROLS and GRACIOUS that were already mentioned by Vicki. In addition, we partner with Procter \& Gamble and academic partners in the Project AeroSafe funded by the German Federal Ministry of Education and Research. The project addresses the suitability of alternative approaches to investigate the potential of fragrances (cosmetic industry), pharmaceutical additives (pharmaceutical industry), and solid particles (chemical industry) to induce adverse effects in the alveolar region of the lung, focusing on inflammatory effects, clearance by macrophages, modulation of barrier function, and the activation of the innate immune system. Based on available in vivo data, AeroSafe aims to find the simplest yet appropriate model, starting with alveolar macrophages, followed by coculture of these macrophages with alveolar epithelial cells, and finally complex models including dendritic cells and endothelial cells.

Dr. Clippinger: What are the critical next steps to achieve global implementation and regulatory acceptance of nonanimal approaches, and what specifically can stakeholders from industry, government agencies, $N G O s$, and academia do to contribute to this progress?

Dr. Stone: I think the easiest, shortest answer is to always make sure they have either human or animal data against which to compare their model. I am not going to use the word "validate," because it is about understanding the limitations of your model. In other words, what you can use your model for and what you cannot use it for. When is it appropriate to make conclusions that are relevant to risk assessment, and when is it not using your particular model? You can make all the in vitro models in the world, but if you have not shown whether or not it is relevant to either human or animal data, then it is very difficult to use it.

\section{Dr. Clippinger: So, a key part of accomplishing that goal is contributing existing data to publicly available databases?}

\section{Dr. Stone: Yes.}

Dr. Landsiedel: I think that is the basis. You have to have a relevant model, and prove that it is relevant. Success stories will lead to successful use of alternative methods for inhalation toxicity. We should identify which effect and what substances we want to assess, and then find the right tests. Then we can achieve something.

I think short-term success examples are grouping strategies, because grouping can easily be guided, aided, and supported by in vitro methodology and becomes a lot stronger than grouping only based on chemical structures, which is impossible for most particles anyway. So, a grouping concept is something we need to have, and we need to define which substances and which effects are the focus. Then we should see success stories rather soon.

Dr. Stone: I would add that most people think about grouping on the basis of a similar physical or chemical property for a substance that you want to understand its risk assessment. But, if you look at European regulations, the advice is that you should group based on a hypothesis. The hypothesis could be something like lung fibers above a certain length will cause lung cancer. And it could include more complicated types of mechanisms. So, it is not just about looking at the chemical or physical characteristics of the substance that you want to test, but relating it to a biological endpoint. That is where these in vitro models become very useful, because to justify your grouping decision, you need some evidence for that. Your in vitro models allow you to test that hypothesis without going into animals.

Dr. Allen: To your question, achieving global harmonization on classification and labeling is a critical need, and more specifically, characterizing in better detail what information regulators actually need to make regulatory decisions.

During the past 12-18 months, we have heard a lot of discussions around starting with the end in mind and connecting the test method developers with end users and regulators in early discussions. Also, the field would benefit by the availability of sources of funding - be it a Small Business Innovative Research Grant or other grant funding strategies-for test method developers and end users to characterize new approaches.

But, probably more than anything else it is critical to have key decision-makers willing to take a chance and step out of the norm, and to understand and appreciate the data that are being developed with nonanimal approaches. Emily very 
correctly identified some of the inertia that we are often up against. So, it is imperative to have folks in key positions being willing to step outside of their comfort zone and consider new information. The approach that Paul outlined is a great example of that. Maybe that approach becomes a case example of a success story that Robert mentioned, which can be built upon and more broadly characterized. It is truly about having people who are willing to take a chance on what could be a better approach.

Dr. Hinderliter: I would add that our in vitro/in silico approach-although meeting one goal of reducing animal testing-is not a shortcut. It is not a way to quickly substitute an in vitro test in place of an in vivo study. In the end, in developing this approach, we have spent more time and effort, but have learned more about our compound. We feel that we have a better, more comprehensive assessment based on what we now know. This should give regulators some reassurance that this in vitro/in silico approach is not less-it is actually more.

Dr. Reinke: Another thing I would add is the importance of making sure the tests are accessible. In other words, to ensure that there is not an extremely high hurdle to bringing a newer test in and getting it up and running in individual laboratories. Many of the tests that are out there, which are on the edge of being acceptable to regulators, might be extremely hard for a smaller laboratory to bring in and use. For example, it would be useful to make some of the proprietary cell lines that are out there more accessible, so anybody who is interested in doing toxicology testing can use them.

Dr. Behrsing: I fully agree, as Dave mentioned, that conservative scientists need to be willing to take a risk a little bit. For many, they are just a bit hesitant to take that risk knowing if something happens because an in vitro assay did not predict something correctly, then there is some liability there. Having decision-makers who understand the capabilities of these nonanimal approaches and have the desire to see the change that is needed is key.

There is also need for protocol standardization. For example, I still see various publications coming out indicating that precision-cut lung slices only have a longevity of a few days - instead of $>28$ days, which is what we and others have seen in the laboratory. In one case, we found that researchers were able to extend their studies from 6 to 12 days-which is when the experiment ended, although they likely could have gone a lot longer-simply by changing the culture medium.

Dr. Allen: It is important to highlight the amount of resources that have been put into these efforts from PETA and from other NGOs interested in coming up with high- quality, science-driven approaches to move the field forward. There are many really nice examples that can be highlighted.

Dr. Reinke: As a final thought, I would like to recognize something that has been implied throughout our discussion, but an advantage of nonanimal methods over traditional methods is that it means a huge reduction in animal use. The massive advantage is that you are reducing animal use, and that is only going to be good in the long run.

\section{Author Disclosure Statement}

No competing financial interests exist.

\section{References}

1. Oomen AG, Bleeker EA, Bos PM, et al. Grouping and readacross approaches for risk assessment of nanomaterials. Int J Environ Res Public Health 2015:12;13415-13434.

2. Burden N, Aschberger K, Chaudhry Q, et al. Aligning nanotoxicology with the 3Rs: What is needed to realise the short, medium and long-term opportunities? Regul Toxicol Pharmacol 2017:91;257-266.

3. Wiemann M, Vennemann A, Sauer UG, et al. An in vitro alveolar macrophage assay for predicting the short-term inhalation toxicity of nanomaterials. J Nanobiotechnology 2016:14;16.

4. Interagency Coordinating Committee on the Validation of Alternative Methods. A Strategic Roadmap for Establishing New Approaches to Evaluate the Safety of Chemicals and Medical Products in the United States. January 2018. https:// ntp.niehs.nih.gov/pubhealth/evalatm/natl-strategy/index.html (last accessed Mar. 12, 2018).

5. Clippinger AJ, Allen D, Jarabek AM, et al. Alternative approaches for acute inhalation toxicity testing to address global regulatory and non-regulatory data requirements: An international workshop report. Toxicol In Vitro 2018:48;53-70.

6. Strickland J, Clippinger AJ, Brown J, et al. Status of acute systemic toxicity testing requirements and data uses by U.S. regulatory agencies. Regul Toxicol Pharmacol 2018:94;183-196.

7. Behrsing H, Hill E, Raabe H, et al. In vitro exposure systems and dosimetry assessment tools for inhaled tobacco products: Workshop proceedings, conclusions and paths forward for in vitro model use. Altern Lab Anim 2017:45;117-158.

Address correspondence to: Dr. Amy J. Clippinger

Director PETA International Science Consortium Ltd. Society Building 8 All Saints Street London 9RL United Kingdom

E-mail:amyjc@piscltd.org.uk 